\title{
Sensitivity Enhancement of Heterocore Macrobend Fiber Optics by Adding a ZnO Film
}

\author{
Noor Azie Azura Mohd Arif $\mathbb{D}^{1},{ }^{1,2}$ Dilla Duryha Berhanuddin, ${ }^{2}$ and Abang Annuar Ehsan $\mathbb{i D}^{2}$ \\ ${ }^{1}$ Centre for Pre-university Studies, Universiti Malaysia Sarawak, Kota Samarahan 94300, Sarawak, Malaysia \\ ${ }^{2}$ Institute of Microengineering and Nanoelectronics, Universiti Kebangsaan Malaysia, Bangi 43600, Selangor, Malaysia \\ Correspondence should be addressed to Noor Azie Azura Mohd Arif; manaazura@unimas.my and Abang Annuar Ehsan; \\ aaehsan@ukm.edu.my
}

Received 16 January 2021; Accepted 13 April 2021; Published 3 May 2021

Academic Editor: Sulaiman W. Harun

Copyright (c) 2021 Noor Azie Azura Mohd Arif et al. This is an open access article distributed under the Creative Commons Attribution License, which permits unrestricted use, distribution, and reproduction in any medium, provided the original work is properly cited.

\begin{abstract}
Optical fibers with high sensitivity are in demand due to their great potential in sensor application. Semiconductors, such as $\mathrm{ZnO}$, are good materials. Using them as a second cladding offers opportunities in realizing next-generation multimaterial fiber optics. COMSOL Multiphysics is used to simulate heterocore macrobend fiber optics with the same curvature radius but different values of refractive index and thickness of $\mathrm{ZnO}$ films. The optimum thickness of $\mathrm{ZnO}$ films is identified by determining the loss of optical fibers. Macrobend heterocore fiber optics by adding $\mathrm{ZnO}$ thin film has been established by simulating and interpreting the relationship in terms of transmission and refractive index in the evanescent field. These results will provide a reliable fundamental to guide the performance in practice.
\end{abstract}

\section{Introduction}

Bending in an optical fiber can result in a loss of power output, which means the output light intensity is smaller than the intensity of the light source [1]. As the bend becomes more acute, more light leaks out. Bending can be utilized in an intrinsic optical fiber sensor, and it can be categorized using a modulation principle in terms of shift intensity, phase, or wavelength [2]. Basically, shift intensity can be recognized by detection of attenuation signal output from the optical fiber. Simple attenuation is bending on the optical fiber, which uses only mechanical disorder. The sensitivity characteristics of fiber optics can then be realized. A heterocore fiber also generates attenuation. This condition can be related to evanescent theory, in which propagation light is coupled out of the core road. The use of a heterocore fiber is an inexpensive method compared with removing fiber cladding via a chemical process. Combination of bending and heterocore fiber has already been studied by researchers. Relevant research is important and helpful for scientists to create a suitable application from this.
At present, semiconductor optical fibers are an emerging platform for the fiber optic world and thus become an important class of fiber. With the advancement of technology, nanomaterials play an important role in fabrication sensors. Hence, researchers have studied various semiconductors coated on fiber optics to enhance the sensitivity of current sensor-based fiber optics. Examples are $\mathrm{ZnO}$ materials. The high refractive index and carrier mobility of $\mathrm{ZnO}$ cause it to be a highly attractive material for optoelectronic application [3-7]. From previous research, $\mathrm{ZnO}$ is used for sensitivity enhancement, which increases the interest in $\mathrm{ZnO}$, especially $\mathrm{ZnO}$ coated on fiber optics. Here, we simulate and design a $\mathrm{ZnO}$ film with a small thickness and high sensitivity of fiber optics at $1550 \mathrm{~nm}$. We then determine the correlation between bending in optical fiber sensors and the heterocore effect.

\section{COMSOL Multiphysics Simulation}

The wave optic module of COMSOL Multiphysics has been used for boundary mode analysis and optical loss simulations. Once the geometries are set, refractive indices are 
given to the core and cladding materials. In this simulation, a wavelength of $1550 \mathrm{~nm}$ is used as a source. The specifications for the proposed fiber are provided in Table 1.

Figure 1 is the geometric model on plane $X Y$ in this simulation with Port 1 is the input terminal while Port 2 is the output terminal. Port 2 is a function of detecting the transmission light intensity. In this simulation, $1550 \mathrm{~nm}$ is used as a light source. Region 2 is the macrobend heterocore portion of the evanescent field. A mapped meshing element is used to evaluate the output from this simulation. The governing equation in this problem is the wave equation for the electric field $[8,9]$

$$
E(r)=E_{1}(r) e^{\left(-j k_{1} \cdot r\right)},
$$

for the wave vector set to unidirectional, where $E_{1}$ is a slowly varying field envelope function and approximates the propagation phase for the wave. Inserting this electric field formulation into Maxwell's equation results in the following wave equation for the envelope function $[1,8]$ :

$$
\left(\nabla-j k_{1}\right) \times\left(\left(\nabla-j k_{1}\right) \times E_{1}\right)-k^{2} E_{1}=0 .
$$

\section{Results and Discussion}

3.1. Impact on Refractive Index. Propagation of light in a fiber can form evanescent waves, which can also be transmitted to the external of the fiber. This might be occurred due to the structure of fiber optic such as macrobend fiber optic or heterocore fiber optic. By combination of the structure, it might be formed double values of evanescent waves in evanescent field. Different refractive indices of silica fiber optic with different size of core will impact on influences on the penetration depth and energy of evanescent waves. Due to this situation, it finally changes the output intensity of the optical fiber. Therefore, we can justify the evanescent field characteristics due to the structure and refractive index of fiber optic by analyzing the transmission intensity at the receiving port of the optical fiber. Figure 2 shows the visual map of the electric field along heterocore macrobend fiber optics for different refractive indices. The figure depicts that light intensity is basically concentrated in the core area but has a loss in the evanescent field and bending effect. The values of power flow and electric field at Ports 1 and 2 are depicted in Figure 3. The refractive index is considered the effect on the output. The transmission loss of light is changed by setting different refractive index media and then measuring the output at the receiving end.

From both figures, we can determine that the change in refractive index media can cause a change in energy loss. Power flow is high at Port 2 when the refractive index medium is air $\left(n_{\text {air }}=1\right)$. When the fiber cladding is removed, the power flow along the heterocore macrobend fiber optics is much higher compared with the two others. The total internal reflection is achieved because $n_{\text {air }}$ is much smaller than $n_{\text {core }}$ of fiber optics. Snell's law is obeyed when $n_{\mathrm{ZnO}}$ is larger than $n_{\text {core }}$ of fiber optics. Basically, with the high refractive index of cladding than that of core, the light tends to couple out. Then, the power flow of that situation at Port 2
TABLE 1: List of parameters of fiber optics.

\begin{tabular}{lcc}
\hline Parameter & Single mode fiber $(\mu \mathrm{m})$ & $\begin{array}{c}\text { Multimode } \\
\text { fiber }(\mu \mathrm{m})\end{array}$ \\
\hline Core diameter & 9 & 50 \\
Cladding diameter & 125 & 125 \\
\hline
\end{tabular}

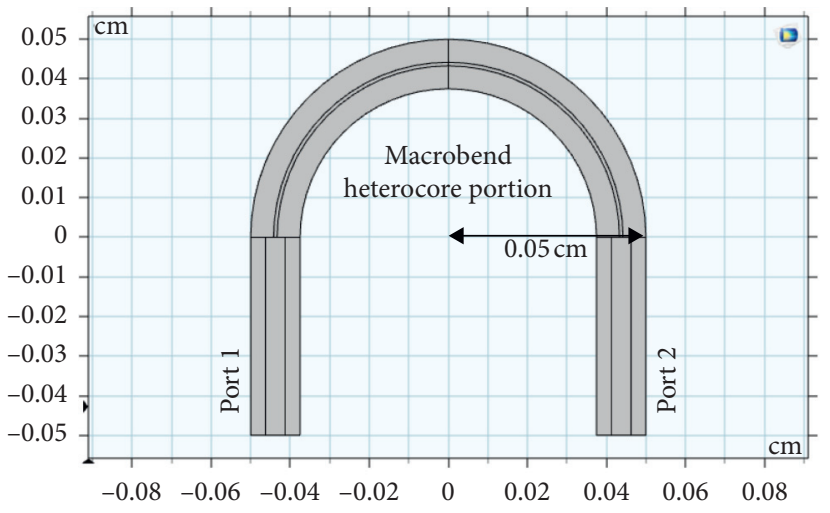

Figure 1: The structure of optical fiber based on bend loss.

is less than the power flow of standard fiber optics. To overcome this phenomenon, the general equation of evanescent wave penetration depth is used $[10,11]$ :

$$
d p=\frac{\lambda}{2 \pi \sqrt{\left(n_{\text {core }}^{2} \sin \theta^{2}-n_{\text {clad }}^{2}\right)}}
$$

where $\lambda$ is the length of the incident wave, $n_{\text {core }}$ is the refractive index of the fiber core, and $n_{\text {clad }}$ is the refractive index of the fiber cladding. Figure 4 shows the penetration depth for different refractive indices of cladding. As the refractive index increases, the penetration depth also increases.

As the foundation principle, light in the fiber optic propagates in a total internal reflection way and extends to the cladding region due to structure and refractive index of fiber optic. Maxwell's equation defines the wave extending to the cladding region as standing wave. If the incident angle is measured from a normal direction at the interface of core and cladding, then the evanescent wave will transmit along the vertical axis and decline exponentially [10]. However, the refractive index of a material impacts the propagation of light. When light propagates through the evanescent area, its energy will be lost. Thus, the greater the light loss is, the smaller energy the receiver perceives. The characteristics of the evanescent field can be analyzed using the light intensity at the output terminal. The results could be described by the relationship between the evanescent light and the characteristics of the refractive index of fiber optic. For further justification, heterocore macrobend optics with different thicknesses of $\mathrm{ZnO}$ film is simulated. Figure 5 shows the result of this situation. The thickness of the $\mathrm{ZnO}$ film influences the propagation of light along the structure fiber optics. As the $\mathrm{ZnO}$ layer increases, the losses decrease. This result can be related to the tunneling optic properties of the refractive index. It can be proven by previous research [12], 


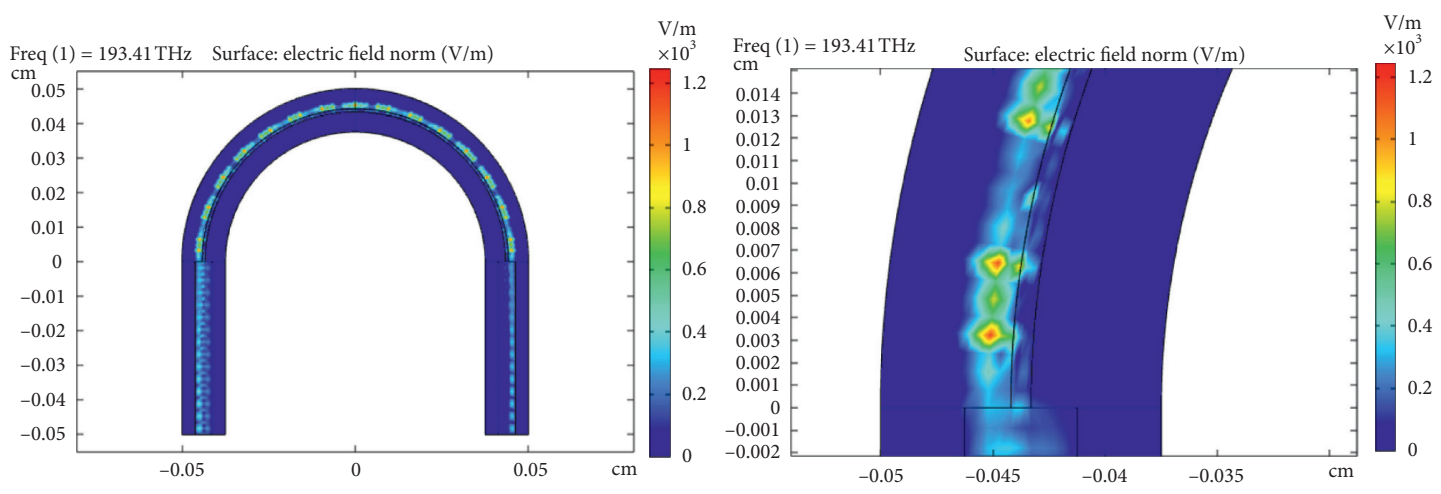

(a)
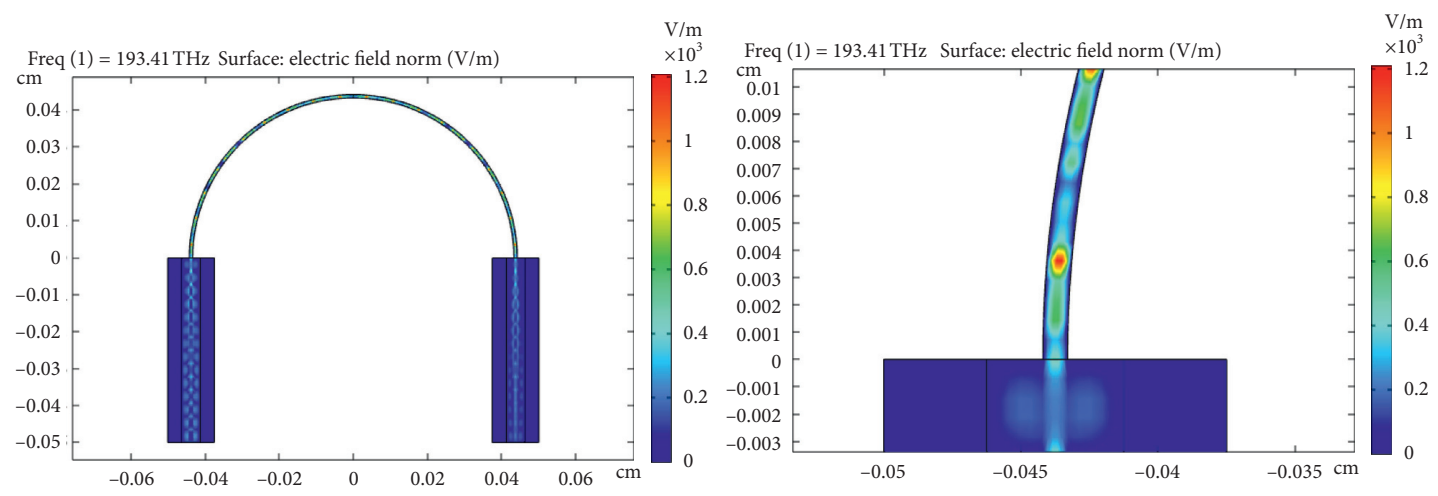

(b)
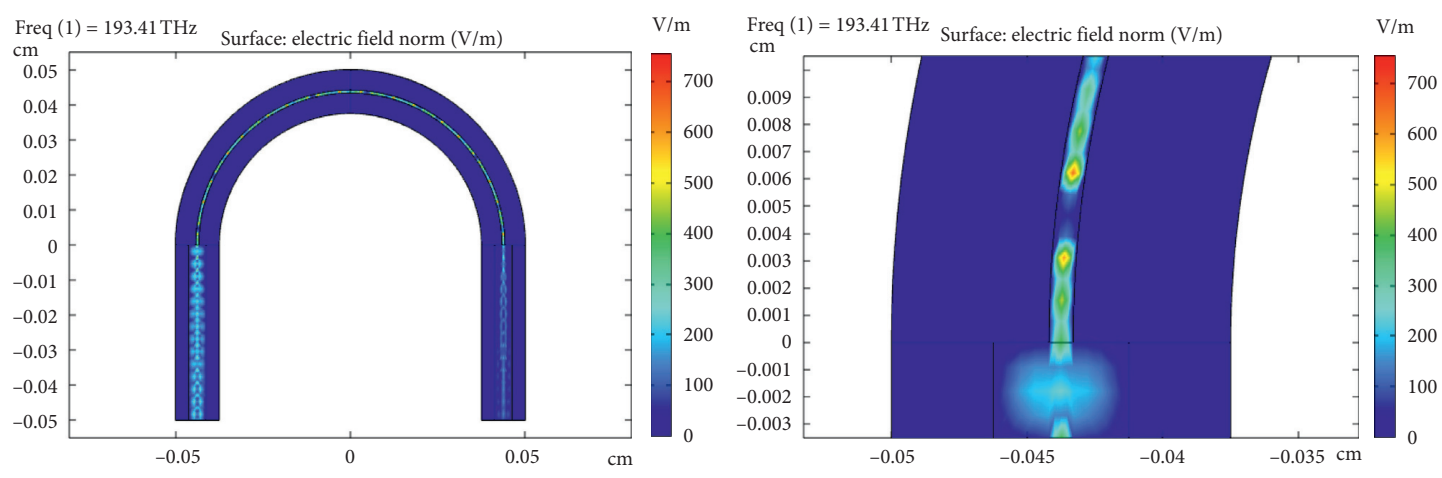

(c)

Figure 2: The electric field propagating along the macrobend heterocore of fiber optic when the refractive index of cladding is (a) $n_{\text {silica }}=1.4612$, (b) $n_{\text {air }}=1$, and (c) $n_{\mathrm{ZnO}}=1.709$.

which mentioned that the energy of $\mathrm{ZnO}$ films decreases as the film thickness increases. The decrease in energy with the increment in film thickness is attributed to the improvement in film quality. Light trapping occurs in media of high refractive index. Light inside a medium of large refractive index is often difficult to be extracted into air, especially if the medium surface is parallel. This phenomenon occurs because certain light undergoes multiple total internal reflection without extraction into air.

3.2. Impact on the Thickness of $\mathrm{ZnO}$ Film. The electric field propagates along the structure of fiber optics with added $\mathrm{ZnO}$ film on the macrobend heterocore portion, as shown in
Figure 6. To determine the optimum fiber geometry, the propagating light is then simulated as a function of $\mathrm{ZnO}$ film thickness. The result is shown in Figure 7. A low loss starting from $5.0 \mu \mathrm{m}$ exists, and the loss increases at the smallest thickness of the $\mathrm{ZnO}$ film because the evanescent wave extends into the cladding region. The effect of introducing additional second cladding is significant in terms of reducing optical propagation losses without an etching process by using a chemical reagent or a costly process. $\mathrm{ZnO}$ has a more significant effect in reducing the loss because the refractive index difference is larger compared with that in a silica fiber. Consequently, some light will propagate out to the surroundings along the transmission. The refractive index causes relative tunneling with increasing $\mathrm{ZnO}$ thickness, as 

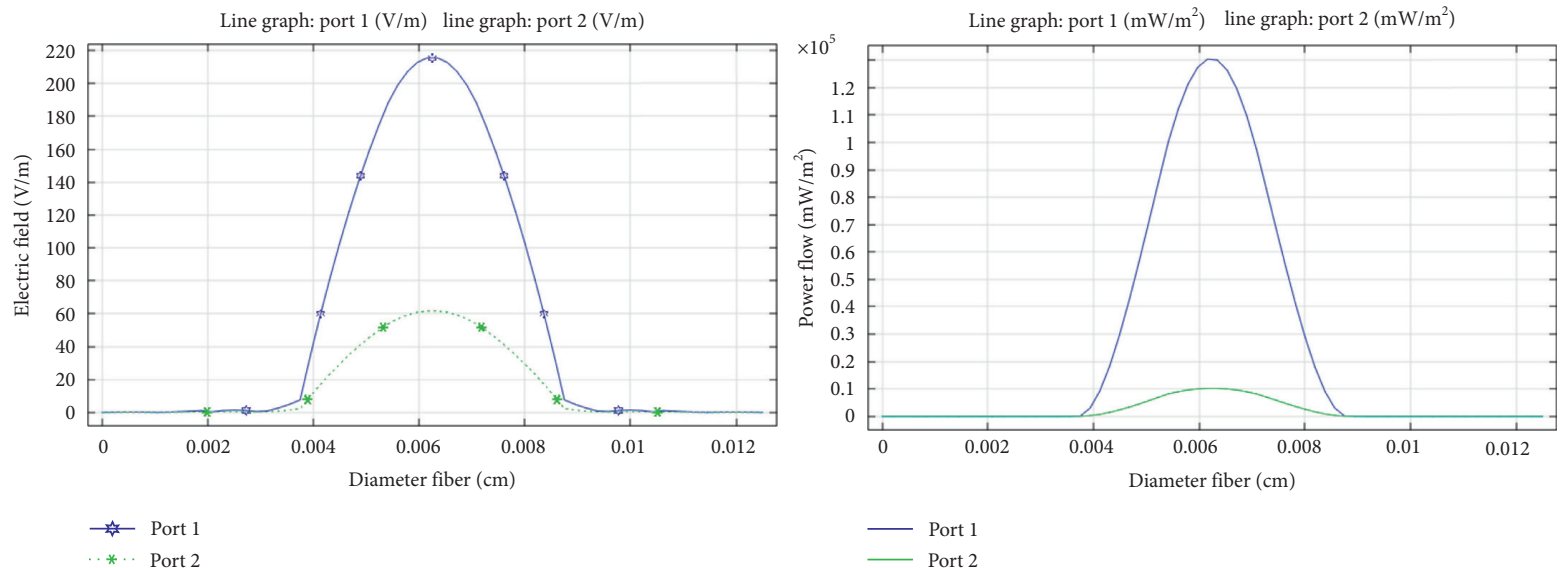

(a)
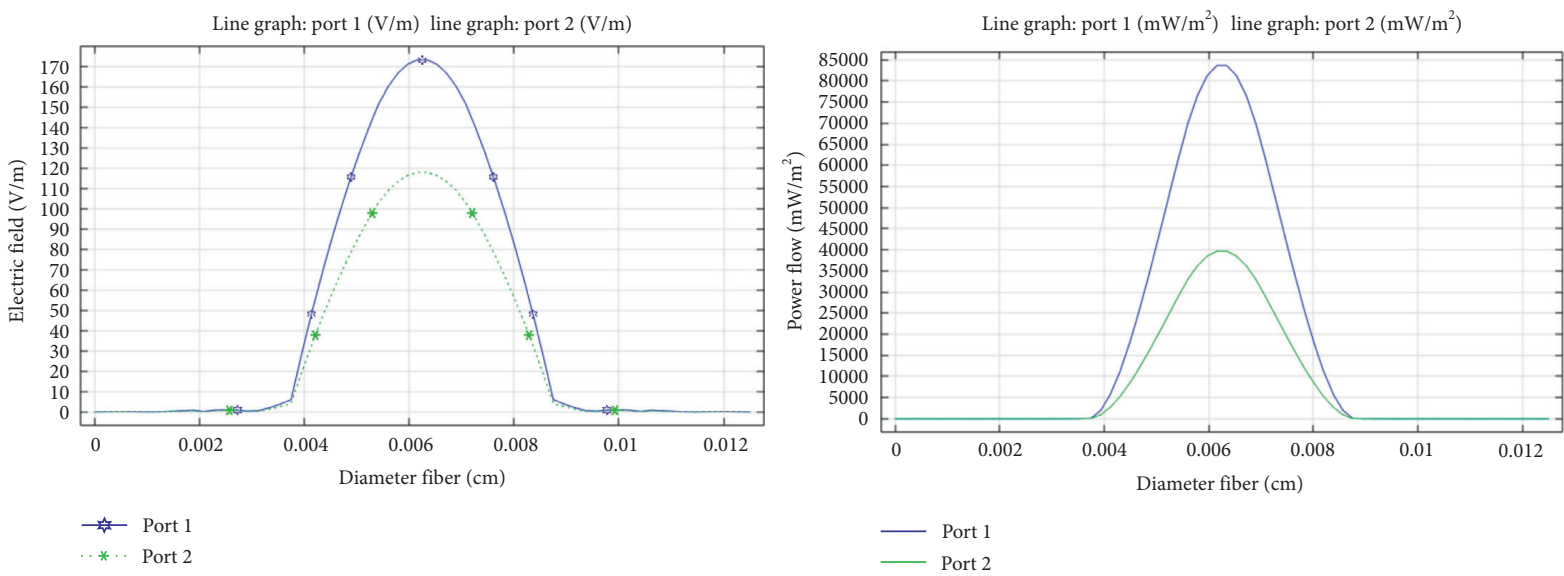

(b)
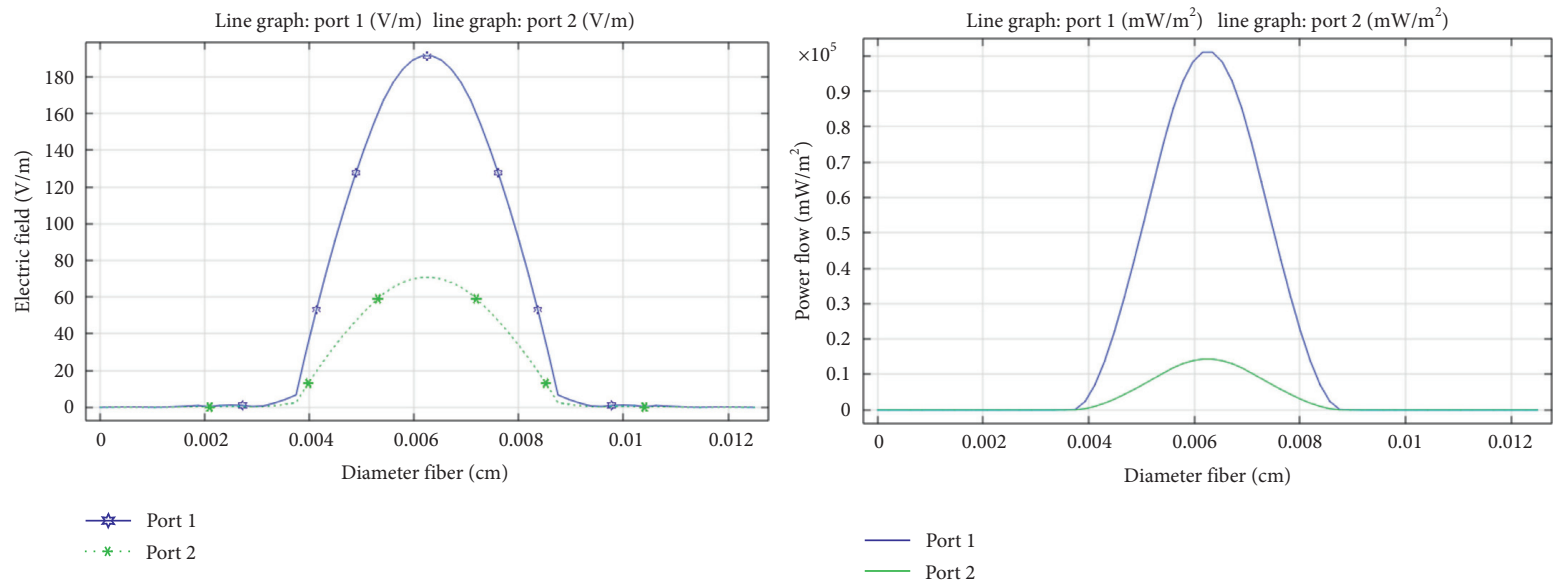

(c)

Figure 3: Graph of electric field and power flow at Port 1 (input) and Port 2 (output) when the refractive index of cladding is (a) $n_{\text {silica }}=1.4612$, (b) $n_{\text {air }}=1$, and (c) $n_{\mathrm{ZnO}}=1.709$.

mentioned in [12]. The relationship between refractive index $n$ and energy gap $E_{\mathrm{g}}$ can be determined as [13]

$$
n^{2}=1+\left[\frac{13.6}{E_{\mathrm{g}}+3.4}\right]^{2} \text {. }
$$




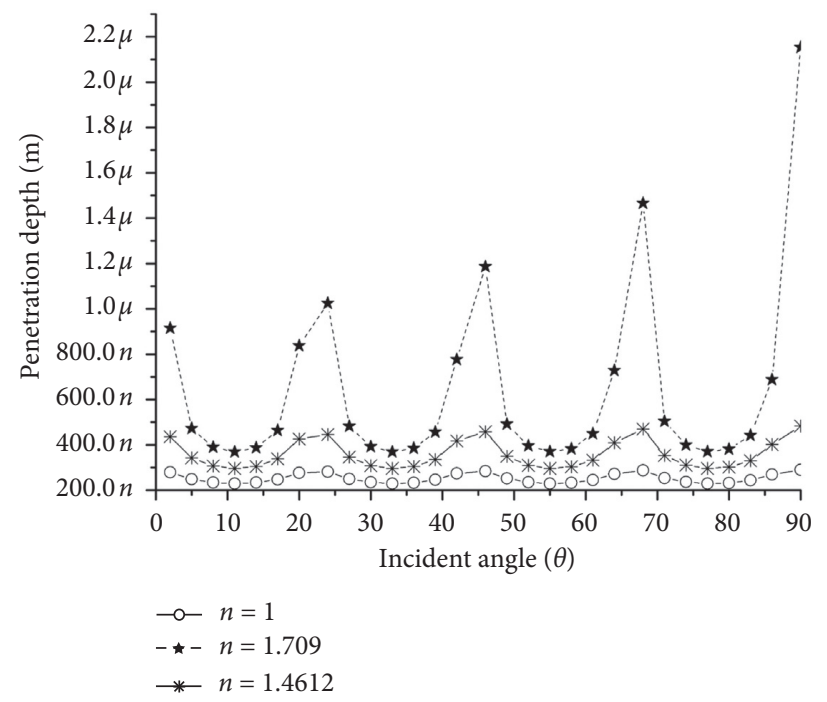

Figure 4: Evanescent wave depth penetration for different refractive indices of cladding.
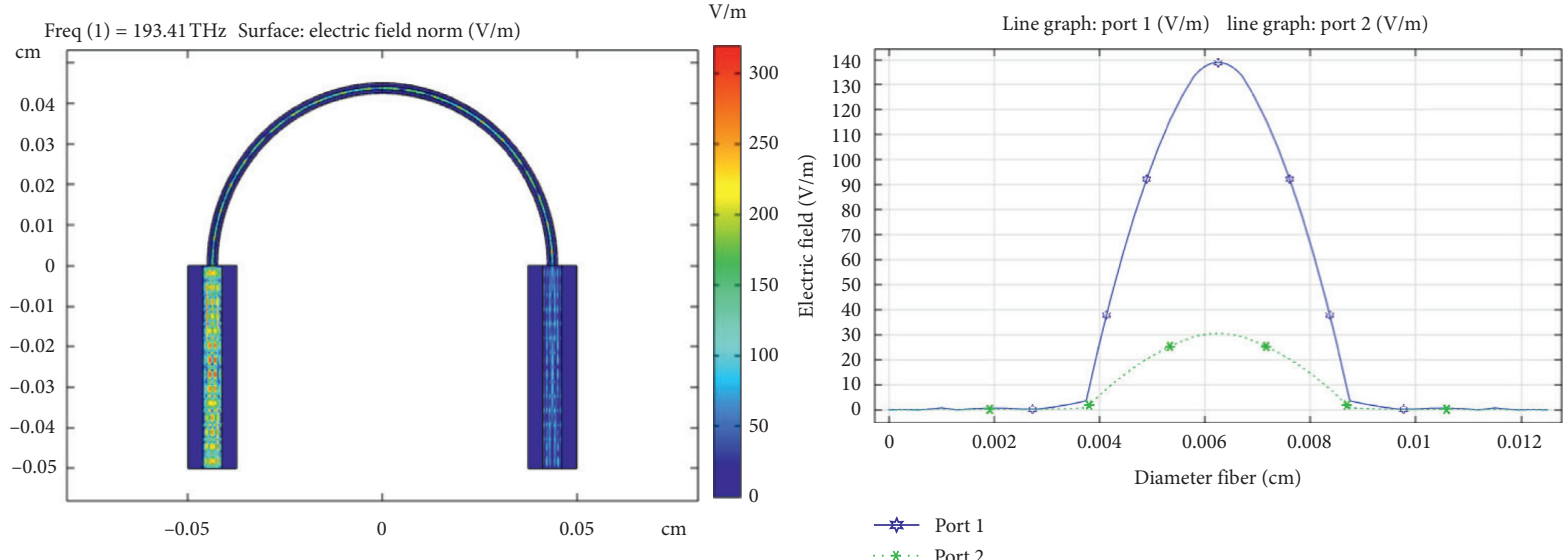

(a)

Figure 5: Continued. 

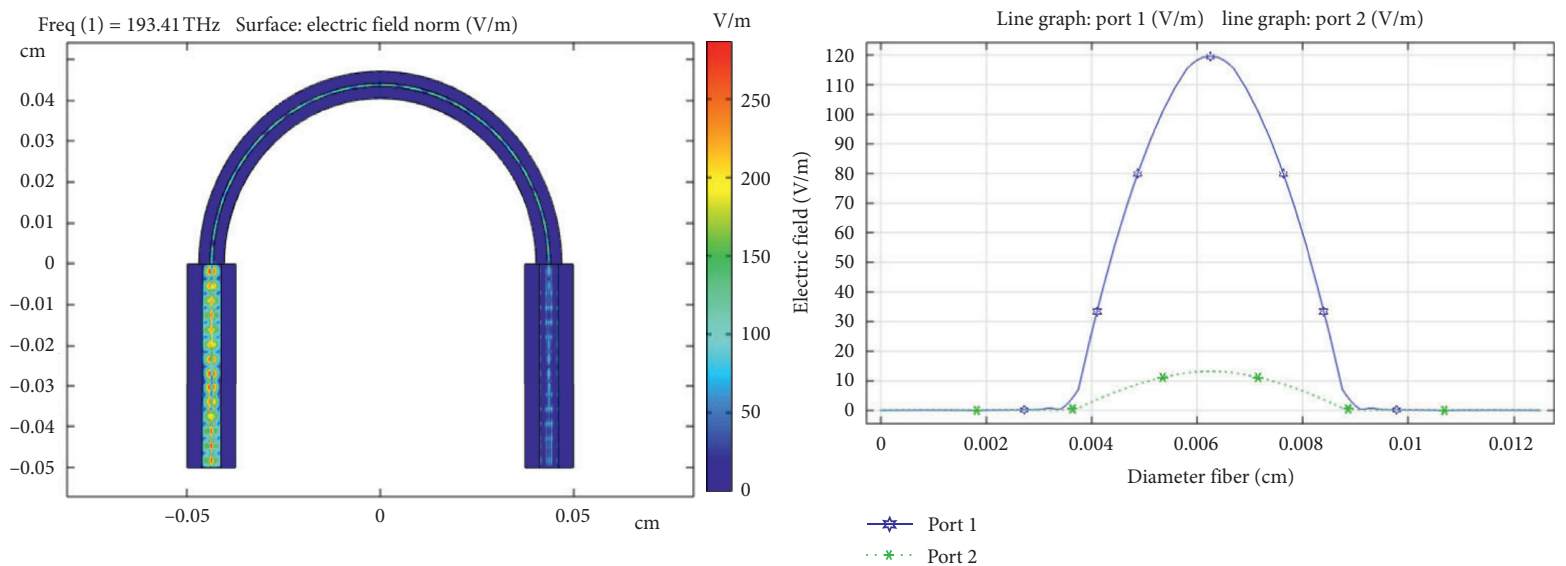

(b)
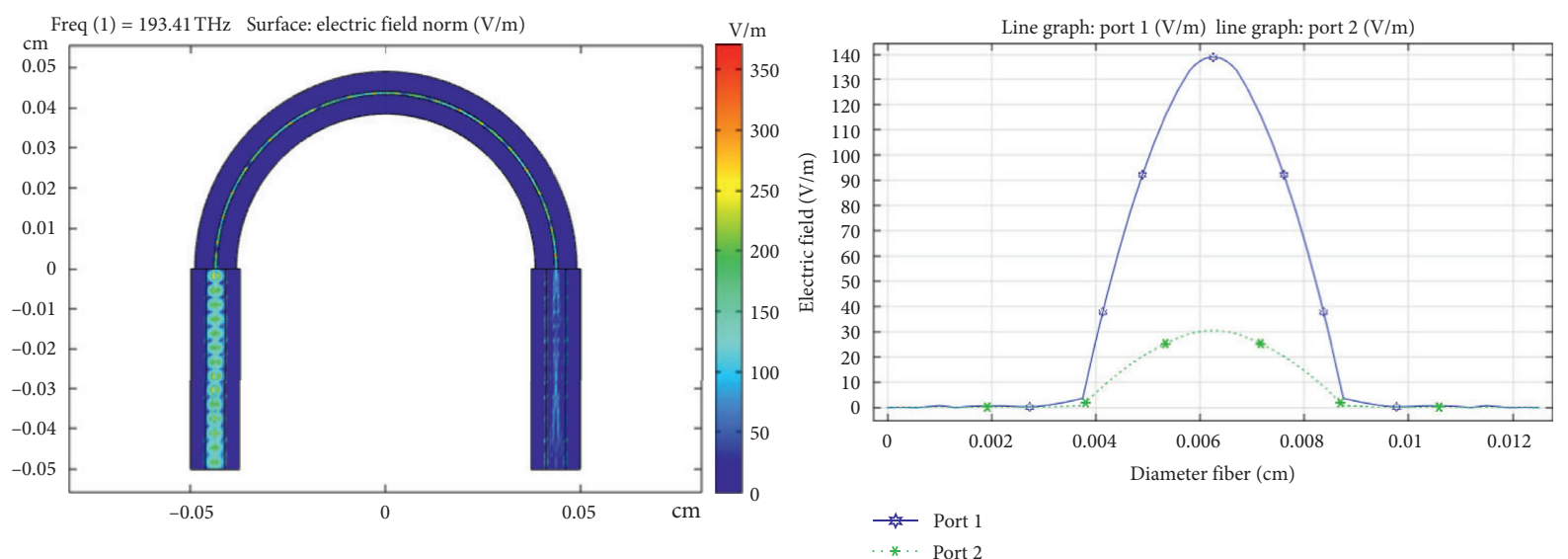

(c)

Figure 5: Electric field and power flow for cladding diameter of $\mathrm{ZnO}$ film. (a) $25 \mu \mathrm{m}$, (b) $65 \mu \mathrm{m}$, and (c) $105 \mu \mathrm{m}$.

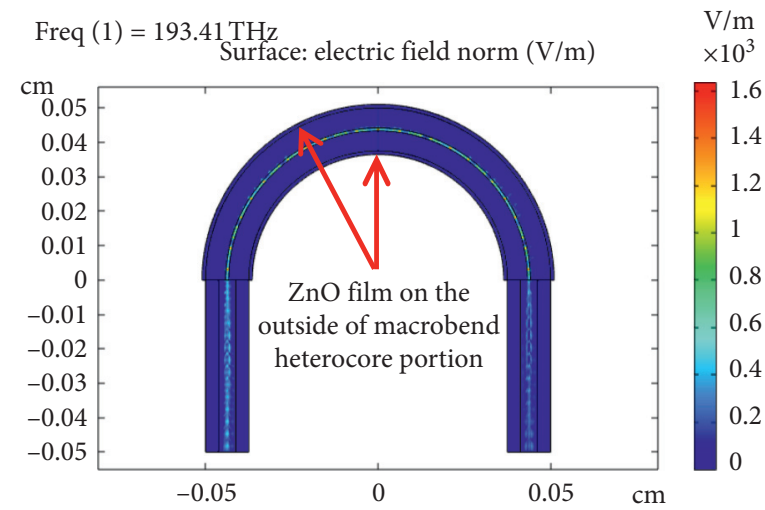

Figure 6: The electric field for different $\mathrm{ZnO}$ thickness.

The transmission output for each structure is shown in Figure 8 . The output clearly shows that, at a certain $\mathrm{ZnO}$ thickness, a high loss occurs. Figure 8(a) illustrates the unclad fiber and $\mathrm{ZnO}$ as a cladding. $\mathrm{ZnO}$ as a second cladding without the original cladding is shown in Figure 8(b). Comparison between these structures indicates 


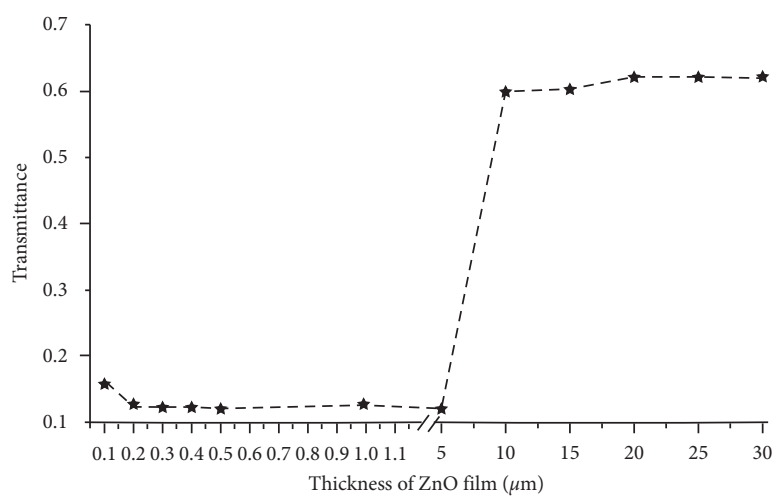

FIgURE 7: Transmission simulated as a function of $\mathrm{ZnO}$ thickness.
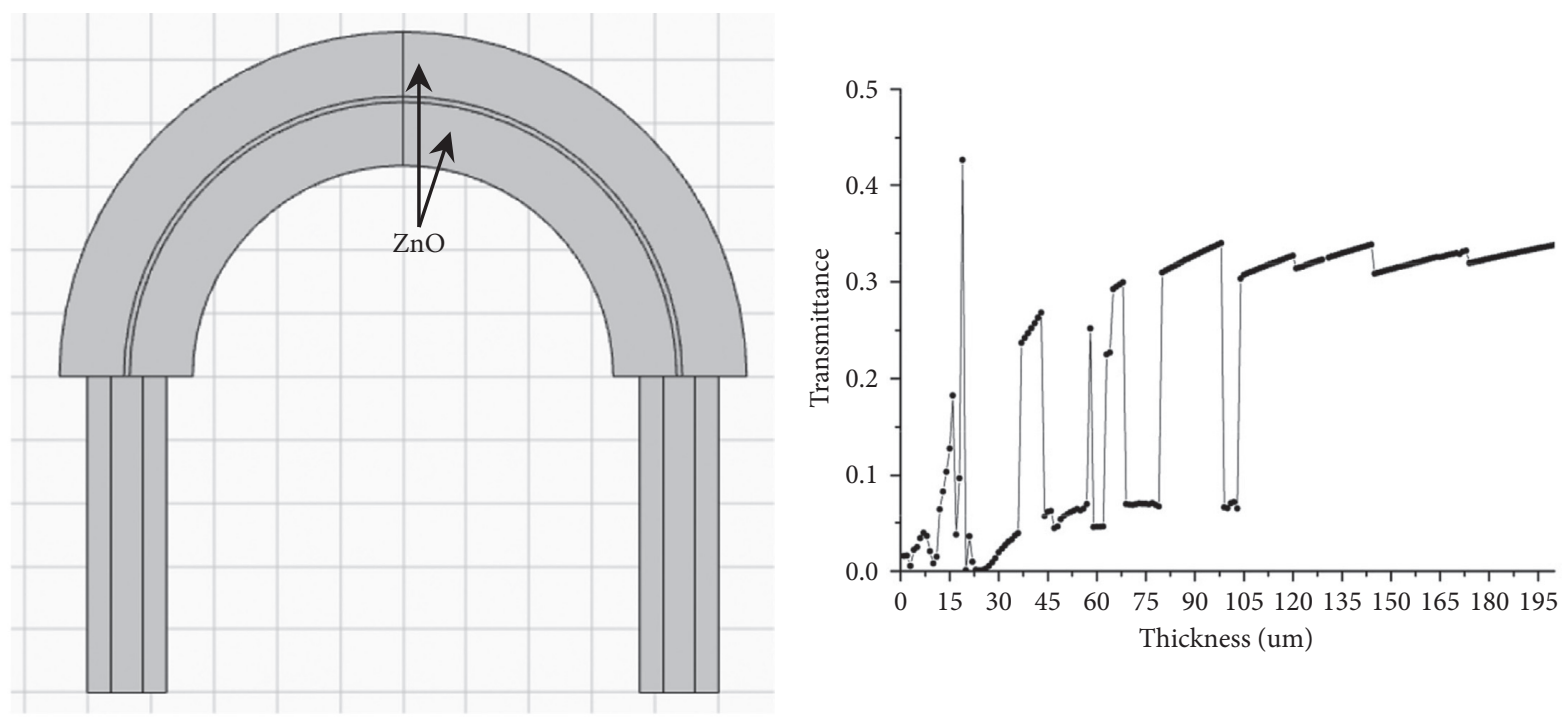

(a)
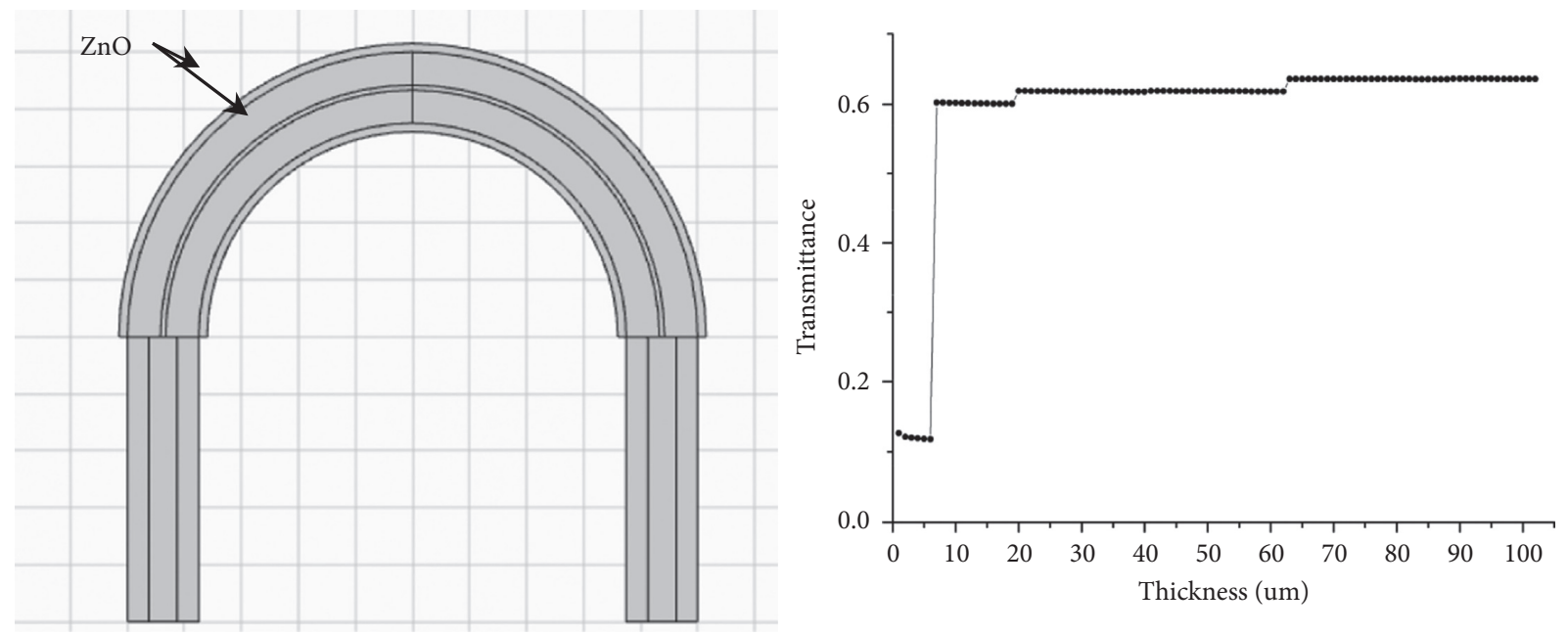

(b)

FiguRE 8: Structure of heterocore macrobend fiber optic with its transmittance graph: (a) $\mathrm{ZnO}$ as a cladding and (b) $\mathrm{ZnO}$ as a second cladding. 
that, for the thickness of $\mathrm{ZnO}$, a high loss appears at less than $10 \mu \mathrm{m}$ without etching silica cladding. By contrast, with $\mathrm{ZnO}$ as a first cladding, the transmission output is unstable until $105 \mu \mathrm{m}$ of $\mathrm{ZnO}$ thickness. However, both simulations showed the similar effective mode index with the value is 1.4819 .

\section{Conclusions}

We have investigated the design of future fiber optics based on a $\mathrm{ZnO}$ optical fiber. The structure and refractive index of its fiber optic proved the impact of evanescent waves in which loss is directly proportional to the refractive value. The thickness of the $\mathrm{ZnO}$ film is varied to optimize fiber performance. The loss is greater when the thickness is less than $10 \mu \mathrm{m}$ for double-cladding fiber optics. This finding can provide a theoretical basis for us to design high-performance heterocore macrobend fiber optics for different purposes in the future.

\section{Data Availability}

The data presented in this study are available on request from the corresponding author. The data are not publicly available due to privacy.

\section{Conflicts of Interest}

The authors declare that they have no conflicts of interest.

\section{Acknowledgments}

The authors are sincerely thankful to Prof Dr Sahbudin Shaari for his proper guidance and supportive discussion. The authors are also thankful to Photonic Lab, Institute of Microengineering and Nanoelectronics (IMEN), UKM, for providing facilities. Financial support from the Ministry of Higher Education (MOE) and Universiti Malaysia Sarawak is gratefully acknowledged.

\section{References}

[1] N. A. A. Mohd Arif, D. D. Berhanuddin, and A. A. Ehsan, "Design parameters of fiber-optic bend for sensing applications," International Journal of Nanoelectronics and Materials, vol. 13, pp. 107-112, 2020.

[2] S. N. Hidayah, R. H. Abdul, H. Haroon, M. Z. A. Suhaila, and S. H. S. M. Fadzullah, "Sensitivity enhancement of singlemode-multimode-singlemode fiber optic sensor based on macrobending effect for food composition monitoring," Journal of Physics, vol. 1151, no. 1, 2019.

[3] G. S. Mei, M. P. Sushita, and G. Hedge, "ZnO for performance enhancement of surface plasmon resonance biosensor: a review," Materials Research Express, vol. 7, no. 1, 2020.

[4] S. Azad, R. Parvizi, and E. Sadeghi, "Tapered optical fiber coated with $\mathrm{ZnO}$ nanorods for detection of ethanol concentration in water," International Journal of Optics and Photonics, vol. 12, no. 2, 2018.

[5] N. F. Idris, M. Q. Lokman, S. W. Harun, H. R. A. Rahim, N. F. Hasbullah, and N. Saidin, "Influence of growth duration to the Zinc Oxide $(\mathrm{ZnO})$ nanorods on single-mode silica fiber," Journal of Telecommunication, Electronic and Computer Engineering, vol. 10, no. 2-7, 2018.

[6] N. Nithya and S. Radhakrishnan, "Effect of thickness on the properties $\mathrm{ZnO}$ thin films," Pelagiaresearchlibrary.Com, vol. 3, no. 6, pp. 4041-4047, 2012.

[7] L. Coelho, D. Viegas, J. L. Santos, and J. M. M. M. De Almeida, "Characterization of zinc oxide coated optical fiber long period gratings with improved refractive index sensing properties," Sensors and Actuators B: Chemical, vol. 223, pp. $45-51,2016$.

[8] Cosmol, Wave Optics Module Users Guide, 2015 COMSOL Multiphysics v.5.2, COMSOL, Stockholm, Sweden, 2015, http://www.comsol.com.

[9] N. A. A. Mohd Arif, D. D. Berhanuddin, and A. A. Ehsan, "2D propagation simulation of variation parameters of U-shape fiber optic," International Journal of Engineering and Advanced Technology (IJEAT), vol. 10, no. 2, pp. 2249-8958, 2020.

[10] L. W. Li and X. H. Sun, "Investigation on the tapered fiber evanescent-field sensor based on the comsol software," in Proceedings of the 2012 Symposium on Photonics and Optoelectronics, Shanghai, China, May 2012.

[11] F. Abdurrahman, N. Arsad, A. Ashrif, A. Bakar, G. K. Beng, and S. Shaari, "Modified multimode polimer optical nanofiber for volatile organic compound sensing in room temperature," Journal of Optoelectronics and Advanced Materials, vol. 21, pp. 313-319, 2019.

[12] A. Bedia, F. Z. Bedia, M. Aillerie, N. Maloufi, and B. Benyoucef, "Influence of the thickness on optical properties of sprayed $\mathrm{ZnO}$ hole-blocking layers dedicated to inverted organic solar cells," Energy Procedia, vol. 50, pp. 603-609, 2014.

[13] P. Hervé and L. K. J. Vandamme, "General relation between refractive index and energy gap in semiconductors," Infrared Physics \& Technology, vol. 35, no. 4, pp. 609-615, 1994. 